\title{
Hearing preservation after removal of small vestibular schwannomas: the role of $A B R$ neuromonitoring
}

\author{
Hussam Abou-Al-Shaar ${ }^{1} \cdot$ Abdullah M. Abunimer $^{2} \cdot$ Timothy G. White $^{1} \cdot$ Amir R. Dehdashti $^{1}$ (D) \\ Received: 13 November 2018 / Accepted: 16 November 2018 / Published online: 23 November 2018 \\ (C) Springer-Verlag GmbH Austria, part of Springer Nature 2018
}

This issue features an article by Mastronardi et al. [7]. The authors evaluated the hearing preservation (HP) after removal of vestibular schwannomas (VS) with a maximum diameter of $<2 \mathrm{~cm}$. Intraoperatively, two case groups were neuromonitored using two different auditory brainstem response (ABR) techniques: classical Click vs LS-CE-Chirp. The authors concluded that Chirp ABR is superior to Click ABR by providing faster feedback, higher wave amplitude, and shorter latency. We commend the authors on their work; however, we would like to shed the light on some points raised by the authors.

Although stereotactic radiosurgery (SRS) might not eliminate the tumor burden and mass effect of VS, several studies have shown promising results related to HP in the management of VS, and various authors suggested that it should not be only considered in recurrent VS cases. Golfinos et al. [5] reported a series of 399 small- and medium-sized VS with a maximum diameter of $\leq 2.8 \mathrm{~cm}$ who were treated with either microsurgery or SRS. Postoperative HP for preoperative class A (based on the American Academy of Otolaryngology-Head and Neck Surgery guidelines) patients was achieved in $14.3 \%$ of microsurgery cases compared with $42.9 \%$ of SRS cases $(p<0.05)$. Serviceable hearing was preserved in $42.8 \%$ of microsurgery cases compared with $85.7 \%$ of SRS cases $(p<0.01)$. This large difference between the two groups should be considered when deciding the optimal management approach in individualized fashion. Interestingly, SRS for VS within the first 2 years after diagnosis has shown statistically significant superior long-term HP compared with later-onset ( $>2$ years) treatment [1].

This article is part of the Topical Collection on Tumor-Schwannoma

Amir R. Dehdashti

Adehdashti@northwell.edu

1 Department of Neurosurgery, North Shore University Hospital, Hofstra Northwell School of Medicine, Manhasset, NY, USA

2 Department of Neurosurgery, Brigham and Women's Hospital, Harvard Medical School, Boston, MA, USA
Despite the recommendations for intraoperative eighth cranial nerve monitoring during HP VS resection, several cohort studies reported contradictory results of HP when monitoring was employed compared to unmonitored cases. In 1994, Nedzelski et al. [9] retrospectively compared 56 patients who received intraoperative monitoring with direct continuous cochlear nerve action potentials (CNAP) to 20 unmonitored cases. At 1-year follow-up, the rate of HP was significantly higher in the monitored group $(p<0.02)$ compared to the unmonitored group following VS resection. Conversely, the series of Piccirillo et al. [10] showed no difference between monitored and unmonitored patients in term of HP, and the presence of CNAP at the end of surgery was not a predictive of good hearing outcomes. In fact, more than half of their patients who had intact CNAP after tumor removal ultimately developed poor hearing outcome. Thus, the reliability of CNAP in anticipating HP or hearing loss cannot be generalized.

Mastronardi et al. [7] claimed that the use of LS-CE-Chirp $\mathrm{ABR}$ as an intraoperative neuromonitoring technique might be superior to Click ABR by providing faster feedback and shorter latency. In fact, the shorter latency of evoked waves by CE-Chirp in comparison with the classical Click is indeed expected since the onset and offset times of CE-Chirp has been pre-adjusted to give such results [15]. Unlike what the authors implied in their paper, many studies have shown that the enhanced neural synchrony obtained by Chirp would increase ABR amplitude rather than affecting the latency. This has been confirmed by comparing Click to an unmodified Chirp (i.e., without temporal adjustment); the unmodified Chirp produced bigger ABR amplitude but more prolonged ABR latency [4]. Based on this data, it is therefore not justified to compare the latencies between classical Click and CEChirp, as the shorter latency does not indicate the superiority of CE-Chirp over other conventional stimuli. Moreover, with the aid of special software systems, the use of combined ana$\log$ filter and digital filter (WF 25 and WF 50) would enable neurophysiologists to obtain interpretable Click ABR traces in 
$5 \mathrm{~s}$ with the summation of fewer sweeps [8]. This will alert the surgeon even faster compared to LS-CE-Chirp ABR system based on the results reported by Mastronardi et al. [7].

In our experience of 30 patients with small-sized vestibular schwannomas (overall mean tumor diameter $1.7 \pm 0.5 \mathrm{~cm}$ ) managed surgically via the retrosigmoid approach, 20 patients demonstrated statistically significant constant and reliable click ABR "V" wave associated with postoperative hearing preservation [2].

Furthermore, the utilization of ABR as a far-field monitor entails several limitations. The first limitation is that many VS patients demonstrate significant hearing loss preoperatively, which makes it difficult to monitor the eighth cranial nerve intraoperatively in the setting of an already reduced ABR wave amplitude. Additionally, various systemic factors such as hypothermia or hypocarbia might affect the brainstem conductivity and change the ABR waveform $[6,11]$. Therefore, using other neuromonitoring modalities instead or in conjunction with $\mathrm{ABR}$ might be more reliable than the utilization of ABR alone.

Among the different intraoperative neuromonitoring techniques, the CNAP monitoring technique seems to be the best modality to reflect the effect of surgical manipulation on hearing as well as predicting postoperative HP/hearing loss [12]. Yamakami et al. [13] utilized CNAP on 44 VS cases with a maximal diameter of $\leq 1.5 \mathrm{~cm}$ who underwent surgical resection. The authors concluded that CNAP was more reliable and associated with significantly better rates of HP than ABR $(66 \%$ vs $32 \%, p<0.01)$ as an intraoperative neuromonitoring technique. Similarly, Danner et al. [3] compared the efficacy of ABR and CNAP monitoring in 66 patients with VS measuring $\leq 2.5 \mathrm{~cm}$. The authors achieved HP in $41 \%$ of patients using ABR while $64 \%$ of their patients achieved HP with direct monitoring $(p=0.03)$. Furthermore, CNAP is associated with lower rates of intraoperative artifacts compared to ABR. Yamakami et al. [14] reported 22 patients who underwent VS resection via the retrosigmoid approach for HP. They monitored all of their patients intraoperatively with CNAP and ABR simultaneously. During surgery, they noticed that $\mathrm{ABR}$ monitoring was disrupted with severe artifacts by various intraoperative maneuvers and surgical instruments, especially when using the microscope, compared to CNAP. As a result, a distinct ABR "V" wave was only obtained in 9/ 22 patients $(41 \%)$ while it was missed in the rest. In contrast, no significant artifacts were observed in CNAP monitoring group and, hence, a reliable CNAP monitoring without artifacts was obtained in $20 / 22$ patients $(91 \%)$.

However, CNAP still carries inherent limitations. The presence of large VS with multiple vessels enclosing the cochlear nerve might make it difficult to safely localize the proximal portion of the nerve for electrode insertion and optimal monitoring. Finally, the risk of damaging the nerve fibers while placing the electrodes should be taken into consideration.
Therefore, future prospective clinical trials are needed to confirm the ideal neuromonitoring modality for HP during VS resection.

\section{References}

1. Akpinar B, Mousavi SH, McDowell MM et al (2016) Early radiosurgery improves hearing preservation in vestibular schwannoma patients with normal hearing at the time of diagnosis. Int J Radiat Oncol Biol Phys 95(2):729-734

2. Chiluwal AK, Rothman A, Svrakic M, Dehdashti AR (2018) Surgical outcome in smaller symptomatic vestibular schwannomas. Is there a role for surgery? Acta Neurochir 160(11):2263-2275

3. Danner C, Mastrodimos B, Cueva RA (2014) A comparison of direct eighth nerve monitoring and auditory brainstem response in hearing preservation surgery for vestibular schwannoma. Otology \& Neurotol 25:826-832

4. Dau T, Wegner O, Mellert V, Kollmeier B (2000) Auditory brainstem responses with optimized chirp signals compensating basilar membrane dispersion. J Acoust Soc Am 107:1530-1540

5. Golfinos JG, Hill TC, Rokosh R et al (2016) A matched cohort comparison of clinical outcomes following microsurgical resection or stereotactic radiosurgery for patients with small- and mediumsized vestibular schwannomas. J Neurosurg 125(6):1472-1482

6. Grundy BL, Jannetta PJ, Procopio PT, Lina A, Boston JR, Doyle E (1982) Intraoperative monitoring of brainstem auditory evoked potentials. J Neurosurg 57(5):674-681

7. Mastronardi L, Scipio ED, Cacciotti G, Roperto R, Scavo CG. (2019) Hearing preservation after removal of small vestibular schwannomas by retrosigmoid approach: comparison of two different ABR neuromonitoring techniques. Acta Neurochir (in press)

8. Moller AR (1988) Use of zero-phase digital filters to enhance brainstem auditory evoked potential. Electroencephalogr Clin Neurophysiol 71:226-232

9. Nedzelski JM, Chiong CM, Cashman MZ, Stanton SG, Rowed DW (1994) Hearing preservation in acoustic neuroma surgery: value of monitoring cochlear nerve action potentials. Otolaryngol Head Neck Surg 111(6):703-709

10. Piccirillo E, Hiraumi H, Hamada M, Russo A, De Stefano A, Sanna M (2008) Intraoperative cochlear nerve monitoring in vestibular schwannoma surgery: does it really affect hearing outcome? Audiol Neurootol 13(1):58-64

11. Sohmer S, Gold S, Cahani M, Attias J (1989) Effects of hypothermia on auditory-brain and somatosensory evoked response: a model of synaptic and axonal lesion. Electroencephalogr Clin Neurophysiol 74(1):50-57

12. Yamakami I, Ushikubo O, Uchino Y et al (2002) Intraoperative monitoring of hearing function in the removal of cerebellopontine angle tumor: auditory brainstem response and cochlear nerve compound action potential. No Shinkei Geka 30(3):275-282

13. Yamakami I, Ito S, Higuchi Y (2014) Retrosigmoid removal of small acoustic neuroma: curative tumor removal with preservation of function. J Neurosurg 121(3):554-563

14. Yamakami I, Yoshinori H, Saeki N, Wada M, Oka N (2009) Hearing preservation and intraoperative auditory brainstem response and cochlear nerve compound action potential monitoring in the removal of small acoustic neurinoma via the retrosigmoid approach. J Neurol Neurosurg Psychiatry 80:218-227

15. Zakaria MN, Zainun Z, Cheu Lih A (2015) Considerations when analyzing vestibular evoked myogenic potential (VEMP) outcomes elicited by chirp stimulus in healthy participants. J Int Adv Otol 11: 271-272 